\title{
Nutritional surveys: ongoing activities in Europe
}

\author{
BY B. P. M. BLOEMBERG AND D. KROMHOUT \\ Department of Epidemiology, National Institute of Public Health and Environmental Protection, \\ Bilthoven, The Netherlands
}

There are several indications that dietary patterns differ within Europe, and that these patterns change over time. From mortality data it is also known that mortality rates differ between European countries. It is of interest to study these differences in dietary intake in relation to the differences in mortality rates. To be able to study these relations a valid description of the dietary patterns in Europe is necessary.

To get information about the dietary intake in different countries in Europe several sources of information are available:

(1) food balance sheets,

(2) international monitoring projects,

(3) aetiologic studies,

(4) national nutritional surveys.

All these sources of information have their advantages and disadvantages. These will be discussed in the present paper.

\section{FOOD BALANCE SHEETS}

Food balance sheets are published for many countries by the Food and Agriculture Organization (FAO, 1980). These data represent a country's food supply during a reference period. From these data the per capita food supply available for human consumption is calculated. These food supply data are converted into energy and nutrient intakes by using food tables. However, there are several problems associated with food balance sheets:

(1) several assumptions have been made concerning the proportion of the food wasted during preparation, the proportion fed to livestock, production for own consumption etc. To get information on the energy and nutrient intakes in the different countries with these crude food intake data is even more difficult due to differences in quality between the food tables of these countries;

(2) no distinction can be made between sexes or between the different age-groups for the food balance sheet data;

(3) data are available only for whole countries and not for different regions within countries;

(4) only a limited number of nutrients can be studied, e.g. information on different fatty acids is notoriously unreliable;

(5) it is not known what will happen when, in 1992, trade boundaries between the European Community countries will disappear. Then it will become more complicated to collect food balance data on a national level.

Because of all these problems the food balance sheets are of only limited use for study of the differences between the countries. 


\section{INTER.NATIONAL MONITORING PROJECTS}

In two monitoring projects dietary information is collected in different countries in Europe. The first one, the MONICA project, is designed to collect information on trends in cardiovascular diseases and risk factors for these diseases (WHO MONICA, 1988). This project is being carried out in Europe in Belgium, Czechoslovakia, Denmark, Finland, France, Germany, Hungary, Iceland, Italy, Ireland, Poland, Romania, Scotland, Spain, Sweden, Switzerland and Yugoslavia for males and females in the age-range $25-64$ years. For all persons participating in this project, some information is collected about their usual diet. A simple questionnaire provides information about some qualitative aspects of the diet. The questionnaire contains questions about the usual consumption of edible fat, cheese, milk, fish, meat, alcoholic drinks and coffee and tca consumption. These questions give information about the intake of these foods only, but do not describe dietary patterns as a whole.

In some countries participating in the MONICA project more detailed dietary information is being collected, which is, however, not a part of the core protocol. A consequence of this is that the detailed dietary information is not collected in all countries and that the data are not collected in a standardized way. In Europe more detailed information is being collected in Belgium, Denmark, Finland, France, Germany, Ireland, Poland and the USSR for men aged 45-64 years. The methodology used varies from a $24 \mathrm{~h}$ recall to a 3 and $7 \mathrm{~d}$ record method. Because these dietary data are being only collected for males in a limited age-range and with different methodologies, the use of these data for describing differences in food intake patterns within Europe is limited.

The other project is the EURONUT study, carried out in the elderly (de Groot \& van Staveren, 1988). In this study the aim is to explore dietary patterns in the elderly living in different European communities and to formulate hypotheses for follow-up studies. Between 1988 and 1989 random samples of in total 1285 men and 1305 women born between 1913 and 1918 were examined in nineteen centres in twelve countries in Europe (Belgium, Czechoslovakia, Denmark, France, Greece, Hungary, Italy, the Netherlands, Norway, Poland, Portugal, Spain). The dietary information was collected using a dietary history method in combination with a $3 \mathrm{~d}$ weighed record method. Much attention was paid to the standardization of the methodology through concerted development of the protocol, prescribed methodology and training sessions. This study is an example of a valid tool to describe differences in dietary patterns between the European countries. However, the number of people examined was small and, therefore, estimates of the mean intake of foods and nutrients are subject to uncertainty. In addition, the conclusions are valid only with respect to elderly people.

\section{AETIOLOGICAL STUDIES}

Within different countries in Europe several aetiological studies were carried out in which dietary information was collected. Because in all these studies different methodologies were used and different groups of people were examined, the results of these studies cannot easily be used to compare the dietary patterns in different European countries. In this section of the paper two studies will be presented that collected or will collect dietary information in different countries in Europe in a standardized way. 
The first study is the Seven Countries Study that was started in the 1960s by Keys and co-workers in seven different countries of which five were located in Europe: Finland, Greece, Italy, the Netherlands, and Yugoslavia (Keys, 1970). For this study males aged 40-59 years at baseline were selected. At the start of the study dietary information was collected in subsamples of the cohorts using the record method in combination with the duplicate portion technique. The food samples were chemically analysed, and the composition of the diet of these subsamples was used as an indicator of the whole cohort. Based on these data, ecological analyses of diet and mortality could be carried out. The advantage of the chemical analyses is that no national food tables were used; instead, the nutrients were analysed directly. The drawback of this method is that it is expensive and, therefore, can be used in small subsamples only.

In three of the five European countries, Finland, the Netherlands and Italy, data on food consumption by individuals were also collected. This was done in the 1970s and recently in 1990. In all investigations, a dietary history method was used. Based on these data, the relationship between diet and disease can be studied in five cohorts in three countries on an individual level in about 3000 men aged 50-69 years in the 1970s and about 1200 men aged 65-84 years in the 1990s. Again, in this study, only men in a limited age-range were examined.

The second study, a prospective study on diet and cancer, is in the planning phase at present. The project is being coordinated by the IARC and will be carried out in seven countries in Europe: France, Germany, Greece, Italy, the Netherlands, Spain and the United Kingdom (Riboli, 1990). In this study a total of about 400000 people will be examined. Of all these persons aged 20-70 years, dietary information will be collected with an extensive food frequency questionnaire. Also biochemical markers of nutritional intake will be measured in blood and urine. In the pilot phase of the project, much attention will be given to the standardization, repeatability and validation of the dietary assessment methods. When this project is carried out it will yield important information about differences in dietary intake between the participating countries. Due to the use of biomarkers and a reference method, the validity of the comparison between the different countries will be increased.

\section{NATIONAL NUTRITIONAL SURVEYS}

In different countries in Europe, national nutritional surveys are carried out. Some of these surveys are based on households, others on individuals. Also in these surveys there are large differences in methodology and in the assessment of nutrient intake. The advantage of these surveys is that the group studied is representative of the whole population in the country. In this section of the paper the nutritional surveys in the United Kingdom, Germany, Sweden and the Netherlands will be discussed.

\section{United Kingdom}

The National Food Survey in the United Kingdom was started in 1940, and in 1950 in its present form (Ministry of Agriculture, Fisheries and Food, 1987). It is carried out annually in about 7500 households in different regions of the United Kingdom excluding Northern Ireland. Data on food purchases are collected on a household basis by the housewife, who keeps a logbook for $14 \mathrm{~d}$. The information is collected and checked by an 
interviewer, and the data are coded and entered into the computer. No information is available on the intake of the different individuals in the household. Only the composition of the household is known. Information is collected about the number of meals consumed outside, but no information is collected concerning the composition of these meals. Also no information is available on the consumption of alcoholic drinks, soft drinks and candies. The nutrient intake is assessed by several assumptions concerning the amount of waste and the consumption by each member of the household. The results of the surveys are reported the next year in the annual report. Individual data are, however, not available.

\section{Germany}

In Germany, there have been several investigations of the food intake pattern of the population. From 1985 to 1988 , a large nutritional survey was conducted in the former West Germany including a representative sample of 25000 males and females. The survey was household based and the information was collected using a $7 \mathrm{~d}$ record method. In 1991 the first results were available, but individual data are, however, not yet available. A nutritional survey was also a part of the National Health Survey carried out in 1985, 1988 and 1991 in about 5000 males and females in the former West Germany. The methodology used was an interviewer-administered food frequency questionnaire. In a subsample of the respondents an automated dietary history method was also used. The data of 1985 and 1988 are now available on public-use files. At the end of 1991, a similar examination will take place in 2000 persons living in the former East German states (L. Kohlmeier, personal communication).

\section{Sweden}

Until 1989 in Sweden, traditional Household Budget Surveys were carried out. In these traditional household budget surveys information was collected only on the acquisition of foods to the household during a certain period of time. No data on foods eaten outside the household and no data on the consumption of foods by the individual household members were available. In 1989 a new type of survey was undertaken by the Statistics Sweden and the National Food Administration. In this survey a representative sample of 3000 persons aged 0-74 years was selected. The household to which the selected person belonged was included in the survey. To collect the information on household food purchases, a 4-week record of these purchases was kept by the person responsible for the household. To get information about the individual food consumption, a precoded $7 \mathrm{~d}$ record of food intake was kept by the selected household member. In this way it was possible to collect food consumption data at both the household and the individual level (W. Becker, personal communication).

\section{The Netherlands}

In 1987 and 1988 a national nutritional survey was carried out in the Netherlands in about 2500 households forming a representative sample of the Dutch population (Netherlands Ministry of Welfare, Public Health and Culture, 1988). For each member of the household individual food consumption data were collected during two consecutive days 
with a $2 \mathrm{~d}$ dietary record method. In total, information of about 6000 persons was collected. Information on foods eaten outside the household was also collected. The nutrient content of the foods was calculated with the use of the national food table. Next year the data will become available on public-use files. A new survey is planned for 1992.

\section{CONCLUSION}

Different kinds of activities are taking place in Europe to describe dietary intake. Most activities are either concentrated on specific groups (e.g. only males, or specific age-groups) or concentrated on a specific country. Because of differences in methodology it is only possible to get an impression of the dietary patterns in Europe. It is, however, not possible to obtain an accurate picture of the dietary patterns. Therefore, these data are of limited value for a European nutritional surveillance system. To get a valid picture of the differences in dietary intake patterns of the different European countries more attention should be given to standardization of the dietary surveys. This will enhance the value of the data collected. However, in several countries nutritional surveillance activities have already started, and one of the purposes is to measure time trends in dietary intake. Therefore, it is often difficult to adapt the methodology to the 'European standards', because changing the methodology might hamper the comparisons within countries over time. One way to overcome this problem is to combine the old methodology with the new one, as was done in Sweden in 1989. Another possibility is to validate the different methods by conducting specific validation studies.

\section{REFERENCES}

de Groot, L. \& van Staveren, W. [editors] (1988). Nutrition and the Elderly. Euro-Nut Report 11. Wageningen: EURONUT.

FAO (1980). Food Balance Sheets and per capita Food Supplies. Rome: Food and Agriculture Organization of the United Nations.

Keys, A. (1970). Coronary heart disease in seven countries. Circulation 41, Suppl. 1, 1-211.

Ministry of Agriculture, Fisheries and Food (1987). Household Food Consumprion and Expenditure. London: H.M. Stationery Office.

Netherlands Ministry of Welfare, Public Health and Culture (1988). Wat eet Nederland. Resultaten van de voedselconsumptiepeiling 1987-1988 (in Dutch). Rijswijk: Ministry of Welfare. Public Health and Culturc.

Riboli, E. (1990). The IARC programme of prospective studies on nutrition and cancer. Recent progress in research on nutrition and cancer 1990, 189-204.

WHO MONICA Project (1988). The World Health Organization Monica Project (Monitoring Trends and Determinants in Cardiovascular Disease): A Major International Collaboration. Journal of Clinical Epidemiology 41, 105-114. 